\title{
Intégration professionnelle des populations d'origine et de nationalité étrangères en Suisse
}

Philippe Wanner

\section{(2) OpenEdition \\ 1 Journals}

Édition électronique

URL : https://journals.openedition.org/remi/269

DOI : $10.4000 /$ remi.269

ISSN : $1777-5418$

Éditeur

Université de Poitiers

\section{Édition imprimée}

Date de publication : 1 mai 2004

Pagination : 33-45

ISBN : 2-911627-36-9

ISSN : 0765-0752

Référence électronique

Philippe Wanner, «Intégration professionnelle des populations d'origine et de nationalité étrangères en Suisse ", Revue européenne des migrations internationales [En ligne], vol. $20-n^{\circ} 1 \mid$ 2004, mis en ligne le 24 septembre 2008, consulté le 16 avril 2022. URL : http://journals.openedition.org/remi/269 ; DOI : https://doi.org/10.4000/remi.269

Ce document a été généré automatiquement le 16 avril 2022.

(C) Université de Poitiers 


\title{
Intégration professionnelle des populations d'origine et de nationalité étrangères en Suisse
}

\author{
Philippe Wanner
}

1 Avec $20 \%$ d'étrangers, la Suisse figure dans le groupe de tête des pays européens d'immigration, loin devant ses voisins, l'Allemagne (8,9\% d'étrangers en 2001), l'Autriche $(9,4 \%)$, la France $(5,6 \%)$ ou l'Italie $(2,2 \%)$. Cette proportion élevée d'étrangers, due à la fois à une très forte immigration et à une politique de naturalisation plutôt restrictive, interroge sur l'intégration structurelle et sociale des populations d'origine étrangère. Récemment, l'immigration en Suisse a été marquée par une diversification des motifs d'arrivée et des pays d'origine des migrants, mais aussi par la reprise de flux migratoires en provenance des pays limitrophes (Allemagne, France et Italie en particulier) et du Portugal. Dans ce contexte, il est utile de mesurer les caractéristiques d'intégration des collectivités étrangères dans le but de formuler des politiques migratoires et de mettre sur pied des mesures d'intégration adaptées à la population migrante ${ }^{1}$.

2 Depuis quelques années, le niveau d'intégration de la population étrangère a donné lieu à de nombreuses études en Suisse (Wicker et al., 2003). Que ce soit dans le domaine de la famille (Wanner et Fibbi, 2002), des enfants d'immigrés (Bolzman et al., 2000), de l'accès à la formation (Mahnig et al., 1999), des comportements démographiques (Wanner, 2001) ou économiques (Flückiger et Ramirez, 2003), les comportements d'intégration des immigrés représentent des questionnements prioritaires dans la recherche sur la migration.

3 La plupart de ces études ont décrit, dans l'optique de mettre en évidence des inégalités ou des spécificités de vie, les caractéristiques de deux groupes: les «Suisses »- la population de référence - et les «Étrangers », ces derniers pouvant être analysés conjointement ou répartis selon la nationalité. Or, il nous paraît intéressant de mesurer l'intégration sur le marché du travail en faisant non seulement référence aux caractéristiques des personnes de nationalité étrangère, 
mais à celles des personnes d'origine étrangère. Constituant le lien entre ces deux dimensions, la naturalisation joue un rôle charnière. Le sens symbolique ou pratique de la naturalisation, et le rôle de celle-ci sur l'intégration, est en effet au cœur du débat sur la citoyenneté. Les comportements des Suisses d'origine étrangère présentent alors un intérêt élevé, en particulier dans un pays où, parmi les Suisses, on dénombre une proportion proche de $10 \%$ d'origine étrangère (Haug et al., 2002).

4 Dans ce contexte, l'objectif de cet article est de mesurer les caractéristiques d'intégration des communautés issues de l'immigration, avant et après la migration. Un encadré présente le Panel suisse des ménages (voir annexe), qui a été utilisé dans cette étude, ainsi que les méthodes statistiques; Dans cet article sont successivement décrites les principales dimensions socio-démographiques des groupes définis selon l'origine (première partie) et mesurées les caractéristiques d'intégration sur le marché du travail de ces groupes en fonction du niveau de formation achevé, du chômage et de la position dans l'entreprise (deuxième partie). On discutera enfin la portée des résultats obtenus.

\section{Composition de la population d'origine étrangère}

5 Le tableau 1 distribue les individus ayant répondu à l'enquête de 1999 selon la nationalité actuelle et la nationalité des parents à la naissance. En ce qui concerne la nationalité actuelle, $81,8 \%$ des personnes interrogées sont de nationalité suisse: parmi ce chiffre, on compte $6,4 \%$ de personnes déclarant une deuxième nationalité et 75,4 \% ne déclarant détenir que la nationalité suisse. Parmi les 19,2\% d'étrangers, l'Italie, la Péninsule ibérique, et les autres nationalités de l'Espace économique européen (en particulier Allemagne, France, Autriche), sont les nationalités ou groupes de nationalités les plus fréquemment représentées en Suisse.

Tableau 1 : Répartition de la population selon la nationalité actuelle et celle des parents à la naissance

\begin{tabular}{|c|c|c|c|c|c|c|}
\hline & \multicolumn{2}{|c|}{ Nationalité actuelle } & \multicolumn{2}{|c|}{$\begin{array}{l}\text { Nationalité du père à la } \\
\text { naissance }\end{array}$} & \multicolumn{2}{|c|}{$\begin{array}{l}\text { Nationalité de la mère à la } \\
\text { naissance }\end{array}$} \\
\hline & \begin{tabular}{|l|l} 
En \% \\
\end{tabular} & $\mathrm{N}$ & \begin{tabular}{|l|l} 
En $\%$ \\
\end{tabular} & N & En \% & N \\
\hline Suisse & 75,4 & 6361 & 64,1 & 5394 & 61,4 & 5153 \\
\hline Double national (Suisse + autre) & 6,4 & 566 & 1,5 & 120 & 4,0 & 335 \\
\hline Italien & 5,9 & 277 & 7,8 & 461 & 7,6 & 447 \\
\hline Espagnol, Portugais & 2,7 & 136 & 2,7 & 153 & 2,8 & 155 \\
\hline Autre nationalité de l'EEE ${ }^{1}$ & 5,9 & 273 & 9,9 & 619 & 10,6 & 684 \\
\hline Reste du Monde 2 & 3,8 & 186 & 5,1 & 321 & 5,1 & 318 \\
\hline Inconnu & & & 8,9 & 731 & 8,6 & 707 \\
\hline Ensemble & 100,0 & 7799 & 100,0 & 7799 & 100,0 & 7799 \\
\hline
\end{tabular}

Source : Panel suisse des ménages 1999.

6 L'importance des flux migratoires en Suisse peut être appréhendée par la distribution de la nationalité des parents à la naissance: parmi la population interrogée, $65,6 \%$ des pères et $65,4 \%$ des mères étaient de nationalité suisse au moment de la naissance (éventuellement double nationaux). Ces chiffres doivent cependant être considérés en tenant compte du fait que plus de $8 \%$ des personnes interrogées n'ont pas déclaré la nationalité des parents à la naissance.

7 Ces informations permettent d'établir un classement des personnes selon le statut d'origine, dont les effectifs et les caractéristiques démographiques sont présentés au tableau 2. Alors que la population d'origine suisse présente une répartition selon le 
sexe relativement équilibrée, les personnes suisses dont les deux parents sont étrangers à la naissance ont une structure beaucoup plus féminine $(68 \%$ de femmes), essentiellement due à loi sur la nationalité qui, jusqu'en 1991, a favorisé la naturalisation des femmes (acquisition automatique du passeport suisse au moment du mariage avec un Suisse). La mortalité différentielle selon le sexe peut aussi expliquer en partie la sur-représentation féminine dans ce groupe, en particulier parmi les personnes âgées de plus de 60 ans. En revanche, les Européens interrogés sont en majorité de sexe masculin, à l'exception des Espagnols et Portugais, chez qui l'on observe une récente féminisation des flux migratoires (Wanner et Fibbi, 2002).

8 La migration concerne en premier lieu les jeunes, ainsi que le démontrent les structures par âge des étrangers. Les Suisses dont les deux parents sont étrangers présentent en revanche une structure par âge marquée par une proportion élevée de personnes âgées (Tableau 2). Ce groupe n'est pas représentatif de la « deuxième génération" des migrants récents en provenance de Turquie ou d'ancienne Yougoslavie, fortement médiatisée. Plus de la moitié des personnes appartenant à cette catégorie ont en effet des parents allemands, français, italiens ou autrichiens et vivent en Suisse depuis plusieurs décennies.

Tableau 2 : Caractéristiques démographiques des personnes classées selon l'origine, 1999 (en \%).

\begin{tabular}{|c|c|c|c|c|c|c|c|}
\hline & \multicolumn{7}{|c|}{ Statut d'origine } \\
\hline & Suisse & \begin{tabular}{|l} 
Suisse, 1 \\
parent \\
étranger
\end{tabular} & \multirow[t]{2}{*}{$\begin{array}{l}\text { Suisse, les } 2 \\
\text { parents } \\
\text { étrangers }\end{array}$} & Italien & \begin{tabular}{|l} 
Espagnol/ \\
Portugais
\end{tabular} & \begin{tabular}{|l|} 
Autre \\
nationalité de \\
rEEE 1
\end{tabular} & $\begin{array}{l}\text { Nationalité du } \\
\text { reste de } \\
\text { rEurope }{ }^{2}\end{array}$ \\
\hline Sexe & & & & & & & \\
\hline Masculin & 48,3 & 47,0 & 32,3 & 59,4 & 48,7 & 54,9 & 54,7 \\
\hline Féminin & 51,7 & 53,0 & 67,8 & 40,6 & 51,3 & 45,1 & 45,3 \\
\hline $\begin{array}{l}\text { Ensemble } \\
\text { Âge }\end{array}$ & 100,0 & 100,0 & 100,0 & 100,0 & 100,0 & 100,0 & 100,0 \\
\hline $14-19$ ans & 9,6 & 0,9 & 9,2 & 8,4 & 10,9 & 1,9 & 17,1 \\
\hline $20-39$ ans & 30,2 & 41,2 & 27,2 & 48,8 & 55,5 & 40,4 & 60,9 \\
\hline $40-59$ ans & 35,8 & 32,9 & 34,5 & 31,3 & 29,7 & 34,3 & 20,0 \\
\hline 60 ans et + & 24,5 & 24,9 & 29,1 & 11,5 & 3,9 & 23,4 & 2,0 \\
\hline Ensemble & 100,0 & 100,0 & 100,0 & 100,0 & 100,0 & 100,0 & 100,0 \\
\hline Etat civil & & & & & & & \\
\hline Célibataire & 28,8 & 27,2 & 19,7 & 27,5 & 32,2 & 25,2 & 30,3 \\
\hline Marié & 57,7 & 56,2 & 59,8 & 61,1 & 57,4 & 61,3 & 61,1 \\
\hline Veuf, divorcé & 13,5 & 16,6 & 20,5 & 11,4 & 10,4 & 13,5 & 8,7 \\
\hline Ënsemble & 100,0 & 100,0 & 100,0 & 100,0 & 100,0 & 100,0 & 100,0 \\
\hline Situation familiale & & & & & & & \\
\hline Seul & 18,2 & 23,8 & 19,0 & 14,4 & 12,5 & 24,0 & 15,6 \\
\hline Monoparental & 4,6 & 5,4 & 6,5 & 3,6 & 8,3 & 5,9 & 3,7 \\
\hline Couple sans enfant & 31,7 & 29,1 & 30,0 & 24,4 & 22,2 & 33,9 & 13,5 \\
\hline Couple avec enfant & 43,1 & 37,8 & 42,2 & 56,8 & 57,1 & 34,6 & 64,0 \\
\hline Autre & 2,5 & 3,9 & 2,3 & 0,8 & 0,0 & 1,6 & 3,2 \\
\hline Ensemble & 100,0 & 100,0 & 100,0 & 100,0 & 100,0 & 100,0 & 100,0 \\
\hline Effectifs & 5395 & 823 & 709 & 277 & 136 & 273 & 125 \\
\hline
\end{tabular}

Source : PSM 1999.

9 Par rapport aux Suisses d'origine, les communautés d'Europe du Sud vivent plus fréquemment dans un ménage familial. Ce sont parmi les ressortissants du reste de l'Europe communautaire que l'on observe une proportion élevée de couples sans enfant. Les Suisses d'origine étrangère se caractérisent pour leur part par une structure familiale proche de celle des Suisses d'origine. En revanche, les Suisses issus d'un couple mixte montrent une forte proportion de ménages individuels et une plus faible proportion de couples avec enfants. 


\section{Intégration structurelle des personnes issues de l'immigration}

10 L'intégration structurelle peut être étudiée selon différents angles (Werner, 1998): le niveau de formation atteint informe sur les ressources disponibles pour le migrant dans l'accès à un emploi intéressant; le risque de chômage et la position dans la profession représentent des dimensions objectives de l'intégration dans l'emploi. Ces dimensions sont analysées dans le présent chapitre.

\section{Niveau de formation}

11 Un déficit de formation s'observe pour les étrangers ressortissants de l'Europe du Sud, voire de l'Europe non communautaire, par rapport aux Suisses d'origine (Tableau 3), ceci même après prise en considération du niveau de formation des parents et de l'âge à l'entrée en Suisse. Pour ces personnes, la probabilité d'achever une formation supérieure est diminuée par trois, comparativement au groupe de référence. Ce déficit de formation diminue pour les Italiens lorsque l'on limite l'analyse aux enfants scolarisés en Suisse, signifiant un processus de convergence dès la $2 e$ génération. Le niveau de formation est en revanche significativement plus élevé chez les Suisses d'origine étrangère.

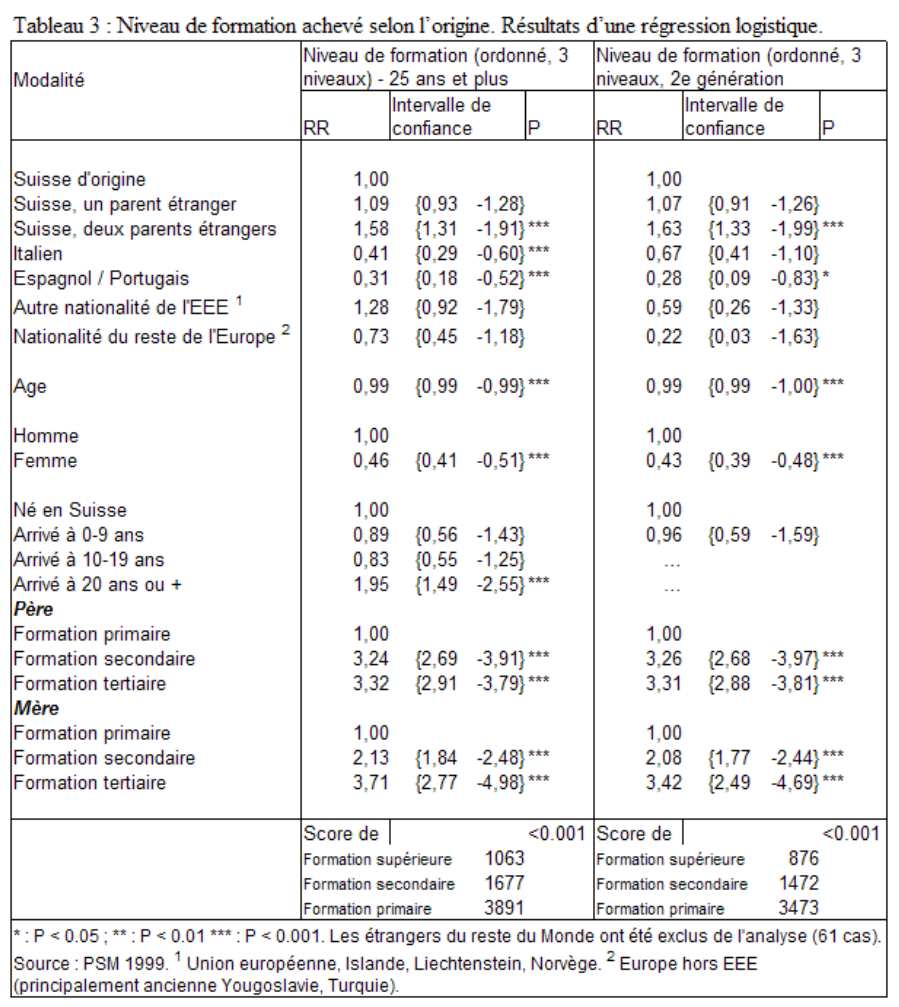

Source : PSM 1999.

\section{Taux de chômage}

12 Au sein de la population active, la probabilité d'être à la recherche d'un emploi au moment du premier passage de l'enquête est plus élevée pour les communautés 
d'origine étrangère par rapport aux Suisses d'origine, à l'exception des Suisses issus d'un mariage mixte. Le risque de chômage des étrangers s'observe après contrôle des facteurs de confusion tels que l'âge ou le niveau de formation. Il concerne en premier lieu les Européens non communautaires. Par rapport aux Suisses d'origine, on observe en outre un risque significativement accru de chômage parmi les personnes dont les deux parents sont étrangers, puisque la probabilité d'être sans emploi est multipliée par 2,6 pour ce groupe.

13 Le taux de chômage élevé des étrangers, en particulier des étrangers non européens, a de nombreuses fois été mis en évidence en Europe. Les facteurs expliquant le risque élevé de chômage semblent ainsi intervenir même après l'acquisition de la nationalité suisse.

\section{Position dans la profession}

14 Le PSM (Panel Suisse des Ménages) met en outre en évidence, après contrôle des variables de confusion, une sur-représentation dans les positions dirigeantes des personnes originaires des pays de la CEE/AELE, hors Europe du Sud et une sousreprésentation des Européens non communautaires (Tableau 4). Ce résultat confirme que la migration en provenance d'Allemagne et de France est axée sur des travailleurs hautement qualifiés. Les Italiens et Espagnols/Portugais ne présentent pour leur part pas un risque significativement inférieur, par rapport aux Suisses, d'exercer une profession dirigeante.

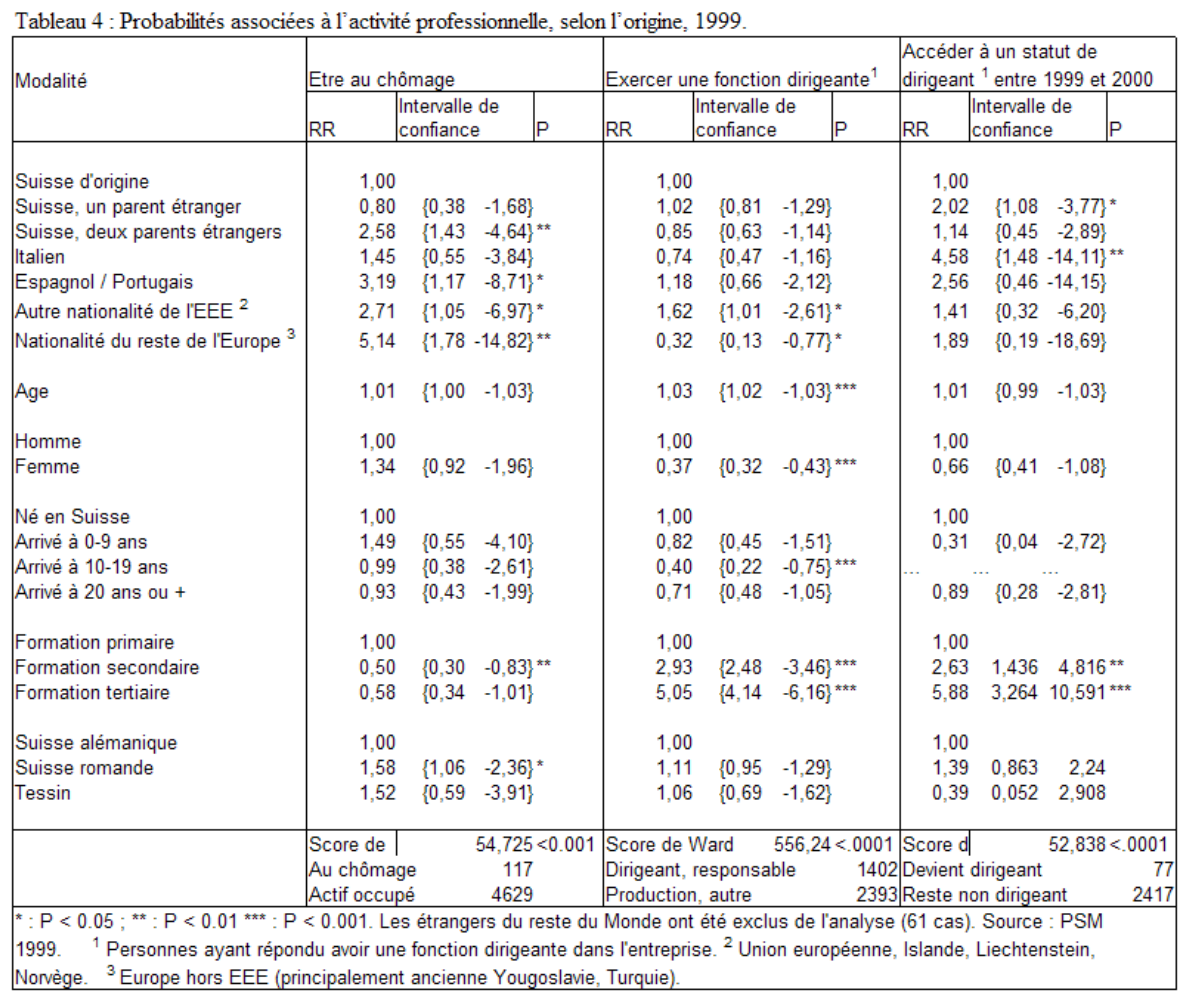

Source : PSM 1999.

15 La mobilité professionnelle a été appréhendée par la position dans l'entreprise en 1999 et en 2000 et par la probabilité d'accès à un poste de dirigeant ${ }^{2}$ entre ces deux dates parmi les personnes non-dirigeantes au cours de la première enquête (Tableau 
4). S'il est clairement établi que la formation et le sexe jouent un rôle essentiel dans la mobilité professionnelle, l'origine intervient également, puisque la probabilité d'accéder à un poste de dirigeant entre 1999 et 2000 est significativement plus importante parmi les personnes de nationalité italienne (risque relatif de 4,58**) et parmi les Suisses dont un parent est de nationalité étrangère (RR 2,02*).

\section{Discussion}

16 Le Panel Suisse des ménages apporte pour la première fois en Suisse des données sur les comportements d'intégration professionnelle des populations issues de l'immigration. L'enquête présente certaines limites, qu'il convient de relever en introduction à cette discussion.

\section{Limite des données}

17 Comme toute enquête, le PSM n'atteint pas l'ensemble de la population immigrée, puisque non seulement les personnes vivant dans des ménages collectifs sont exclues de l'échantillon, mais aussi celles n'ayant pas une liaison téléphonique ou ne parlant pas une des langues nationales utilisées dans l'enquête (allemand, français, italien). Or, les étrangers sont sur-représentés, par rapport aux Suisses, dans les ménages collectifs, représentant $31 \%$ de cette population (Wanner, 2003). Ce sont en particulier les requérants d'asile qui échappent à l'enquête, en raison de leur appartenance fréquente à un ménage collectif. Par ailleurs, même en l'absence d'informations détaillées sur la répartition par origine des personnes ne maîtrisant pas une langue nationale ou ne disposant pas de raccordement téléphonique, on peut penser que l'enquête exclut les résidants de nationalité étrangère présentant les plus faibles niveaux d'intégration. Cet effet de sélection joue un rôle sur l'analyse : les résultats traduisent en effet les caractéristiques des populations issues de l'immigration ayant déjà atteint un niveau d'intégration minimal. Le risque est alors de mettre en évidence des caractéristiques d'intégration meilleures que ce qu'elles ne sont effectivement, en particulier parmi les Européens non communautaires. Pour les autres groupes, le biais est négligeable.

\section{Niveau d'intégration des étrangers sur le marché du travail}

18 Parmi les questions posées de manière récurrente concernant l'égalité des chances des groupes issus de l'immigration, celle de l'intégration professionnelle prend une place essentielle. L'intégration sur le marché du travail signifie pour les étrangers de pouvoir bénéficier d'un même accès aux structures de l'emploi que les autochtones. Dans les faits, les personnes issues de l'immigration rencontrent fréquemment, mais pas systématiquement, des difficultés d'intégration structurelle. En particulier, en période de restructuration et de crise économique, le faible niveau de qualification des membres de certaines collectivités étrangères les expose au risque de perdre leur emploi. Les caractéristiques d'emploi des étrangers - une proportion élevée d'entre eux travaille dans des secteurs sensibles à la conjoncture - pourraient aussi intervenir, tandis que des discriminations sur le marché du travail au moment de l'entrée en emploi ou en cas de licenciement peuvent parfois 
être suspectées, comme d'ailleurs une image négative véhiculée par certains groupes de travailleurs étrangers (Penninx et al., 1994). En outre, des barrières juridiques ou linguistiques à l'emploi s'observent. Des discriminations raciales ou culturelles dont peuvent être victimes certains groupes de migrants peuvent aussi jouer un rôle (Werner, 1998).

19 La discussion des conditions de travail des populations migrantes doit nécessairement être effectuée en se référant au niveau de formation. Haug (1995) avait relevé la sur-représentation, par rapport à la population suisse, des étrangers titulaires d'une formation élémentaire d'une part, et ceux titulaires d'une formation universitaire d'autre part, au détriment de ceux titulaires d'une formation intermédiaire. Ces caractéristiques bimodales se retrouvent dans notre analyse, puisque si un déficit de formation s'observe pour les principales communautés migrantes, les Européens communautaires hors pays du Sud présentent un niveau de formation globalement plus élevé. Cette structure de formation s'explique par la nature des flux migratoires vers la Suisse, qui favorise l'immigration de personnes soit fortement qualifiées, soit peu qualifiées. Elle se traduit par des situations de vie très différentes en fonction de l'appartenance à un groupe de formation, et plus généralement de la nationalité.

20 La prise en compte dans le modèle des principaux facteurs de confusion, dont le niveau de formation des parents, conduit à l'observation que le niveau de formation des étrangers se rapproche progressivement de celui des Suisses pour les personnes ayant grandi en Suisse, avec cependant un déficit qui parait se maintenir pour les Européens du Sud.

21 Différentes études (en particulier Flückiger et Ramirez, 2003) soupçonnent des pratiques discriminatoires à l'égard des étrangers de la part des employeurs. Les résultats obtenus ici confirment l'existence de ces pratiques pour les étrangers de l'Europe non communautaire, mais pas pour ceux de l'Europe communautaire. Elles se traduisent par un risque accru de chômage et une probabilité moindre d'accéder à un poste de dirigeant. Certaines dimensions non précisées dans le modèle (durée d'activité au sein de l'entreprise) peuvent cependant jouer un rôle sur ces variations observées dans l'accès aux postes de direction. La forte mobilité des Italiens entre 1999 et 2000 suggère que les différences pourraient s'estomper progressivement.

\section{Le cas des Suisses d'origine étrangère}

22 Les Suisses d'origine étrangère représentent un intérêt particulier dans la problématique de l'intégration. C'est à partir de ces populations que se conditionne et se reformule de manière dynamique l'identité suisse et pour cette raison leurs comportements sur le marché du travail présente un intérêt dans l'analyse de l'intégration. Comme l'indique leur structure socio-démographique, ces personnes présentent une grande hétérogénéité de vécus migratoires - pouvant être naturalisés ou de parents naturalisés avant leur naissance, nés en Suisse ou arrivés au cours de leur vie - et professionnels. Les résultats présentés, s'ils ne reflètent que des comportements moyens, montrent de manière quasi-systématique que les Suisses d'origine étrangère présentent des caractéristiques de formation et professionnelles s'écartant significativement de celles des Suisses d'origine suisse : un niveau de formation élevé, signe d'une intégration globalement réussie, mais 
aussi un risque important de se trouver au chômage, peut-être expliqué par des attentes élevées vis-à-vis du travail (Poglia Mileti, 1999). Le niveau élevé de chômage remet en question la naturalisation vue comme la phase ultime de l'intégration et suggère le maintien des difficultés à entrer dans le marché du travail et à conserver son poste dans des conjonctures défavorables, au moins pour une partie de ce groupe.

23 Ces différents résultats conduisent à l'observation que l'on ne peut pas catégoriser les «Suisses» et les «Étrangers» selon une approche duale, mais que parmi les Suisses, comme parmi les étrangers, les groupes classés selon l'origine présentent des spécificités à prendre en compte.

24 En définitive, l'analyse des données du PSM fournit quelques éléments empiriques de nature intéressante pour alimenter le débat sur la question des caractéristiques des personnes d'origine étrangère sur le marché du travail. Outre qu'elle apporte des informations originales sur les comportements des Suisses d'origine étrangère, l'enquête confirme les disparités de situations professionnelles observées parmi les différentes collectivités immigrées. Parfois limitée dans la teneur des informations disponibles, puisque des domaines aussi importants que le revenu professionnel ou l'exposition aux risques en fonction de l'origine n'ont pu être analysés, l'enquête suggère clairement la nécessité d'investigations complémentaires dans le but de mieux comprendre les mécanismes d'intégration sur le lieu de travail.

\section{BIBLIOGRAPHIE}

BOLZMAN Claudio, VIAL Marie, FIBBI Rosita (2000) Le processus d'insertion et l'identité des adultes d'origine espagnole et italienne en Suisse. Une comparaison entre les naturalisés et les non-naturalisés, in Pierre Centlivres et Isabelle Girod Éds., Les défis migratoires, Zurich, Seismo, pp. 402-410.

COX Richard D., SNELL Joyce E. (1989) The Analysis of Binary Data, 2e Edition, London, Chapman and Hall.

FLÜCKIGER Yves, RAMIREZ José (2003) Positions hiérarchiques et ségrégation par origine en Suisse in Hans Rudolf Wicker, Rosita Fibbi, Werner Haug Éds., Les Migrations et la Suisse, Zurich, Seismo, pp. 290-307.

HAUG Werner, SCHULER Martin, WANNER Philippe (2002) La dynamique spatiale et structurelle de la population de la Suisse de 1990 à 2000, Neuchâtel, Office fédéral de la statistique, 59 p.

MAHNIG Hans, PIGUET Étienne, WIMMER Andreas (1999) The Integration of Children of Immigrants in Switzerland - Country Report on Secondary Analysis : EFFNATIS - Research project within the TSER Programme of the European Commission, Neuchâtel, SFM.

PENNINX Rinus, SCHOORL Jeannette, VAN PRAAG Carlo (1994) The impact of international migration on receiving countries : the case of the Netherlands, The Hague, Nidi, $240 \mathrm{p}$. 
POGLIA MILETI Francesca (1999) Young people of foreign origin born in Switzerland : Between Invisibility and diversity, in Wrench John, Rea Andrea, Ouali Nouria Éds., Migrants, ethnic minorities and the labour market, integration and exclusion in Europe, Houndmills, Macmillan Press, pp 132-152.

PSM (2001), Vivre en Suisse. Panel suisse des ménages 1999-2003, Neuchâtel, Panel suisse des ménages.

WANNER Philippe (2001) Immigration en Suisse : situation et conséquences démographiques, Neuchâtel, Office fédéral de la statistique, $63 \mathrm{p}$.

WANNER Philippe, FIBBI Rosita (2002) Familles en migration. Familles et migration, in OFAS Éds., Familles et migration, Berne, Office fédéral des assurances sociales, pp 9-49.

WANNER Philippe (2003) Population étrangère et intégration. Résultats du Recensement de la population 2000. Neuchâtel : Office fédéral de la statistique (sous presse).

WERNER H. (1998) L'intégration des travailleurs étrangers sur le marché du travail : France, Allemagne, Pays-Bas et Suède, in Conseil de l'Europe Éds., Les mesures de l'intégration. Strasbourg : Conseil de l'Europe, pp. 173-186.

WICKER Hans Rudolf, FIBBI Rosita, HAUG Werner (2003) Les Migrations et la Suisse, Zurich, Seismo, $566 \mathrm{p}$.

\section{ANNEXES}

\section{Données et méthodes}

Le Panel suisse des ménages a été organisé dans le cadre des activités du Programme Prioritaire « Demain la Suisse » financé par le Fonds national pour la recherche scientifique. Le Panel est une enquête nationale, représentative de la population résidente en Suisse, menée à passages répétés d'intervalles d'une année (PSM, 2001). Le choix des variables incluses dans le PSM a été effectué de manière à couvrir un large éventail de domaines. Les deux premiers passages, réalisés en 1999 et 2000, sont utilisés pour cette analyse. La première vague a concerné 5074 ménages et 7799 personnes. Le deuxième passage a retrouvé 7071 personnes, appartenant à 4425 ménages. Les personnes interrogées étaient tirées au sort parmi la population âgée de 14 ans et plus vivant en ménage privé ou ordinaire. Des pondérations ont été calculées sur l'âge, la nationalité (Suisse, étranger), le sexe et l'état-civil.

Ces données permettent de décrire les populations en fonction de la nationalité actuelle et de la nationalité à la naissance des parents. Différentes catégories de personnes issues de l'immigration ont ainsi été proposées : Étrangers classés selon la nationalité, Suisses dont les deux parents sont d'origine étrangère (naturalisés ou dont les parents ont été naturalisés avant la naissance), Suisses dont un seul parent est d'origine étrangère (enfants de mariages mixtes), par opposition à la catégorie des Suisses d'origine suisse (personnes dont les parents sont de nationalité suisse à la naissance). Cette distinction donne une indication de l'origine des personnes formant la population suisse.

Les caractéristiques d'intégration des populations ainsi définies ont été mesurées à partir d'un modèle statistique de régression logistique. Il s'est agi de mesurer, à l'aide 
de ce modèle, pour différents indicateurs, si les réponses des personnes issues de l'immigration s'écartent des réponses recueillies auprès du groupe de référence des Suisses d'origine.

Le modèle de régression logistique s'apparente à la régression multiple ordinaire (Cox et Snell, 1989). La principale différence vient du fait que certaines caractéristiques observées chez les individus de l'échantillon peuvent être de type qualitatif. C'est en particulier le cas de la variable dépendante (variable à expliquer) qui prend la valeur 0 ou 1 (« oui » ou «non »). Le modèle mesure la probabilité que la variable dépendante soit égale à l'état étudié, en fonction d'un vecteur $\mathrm{x}$ de variables pouvant influencer cette probabilité. On calcule le vecteur bde paramètres reflétant l'impact des variables sur la probabilité, par la formule : $\operatorname{Prob}(\mathrm{Y}=1)=\mathrm{e} ß \mathrm{x} /(1+\mathrm{e} ß \mathrm{x})$. Parmi ces variables figure le statut d'origine de la personne, ainsi que différents facteurs de confusion identifiés comme pouvant intervenir sur les liens entre la variable étudiée et le statut d'origine. Ils sont alors introduits dans le modèle afin d'obtenir des résultats après contrôle de l'effet éventuel des facteurs de confusion. Ceux-ci sont : (1) l'âge, considéré sous une forme continue ; (2) le sexe ; (3) l'âge à l'arrivée en Suisse, distribué en quatre modalités (né en Suisse, arrivé en Suisse entre 0 et 9 ans, entre 10 et 19 ans, ou après l'âge de 20 ans) ; (4) le niveau de formation, en trois modalités selon la typologie classique (formation primaire, formation secondaire, formation tertiaire) ; (5) le lieu de domicile, en trois catégories définies en fonction de la langue parlée (région alémanique, région romande, Tessin). Dans le cas où le modèle s'intéressait à la mesure du niveau de formation achevée, cette variable devient la variable dépendante. On a alors considéré le niveau de formation des parents comme variable contrôlant l'origine sociale.

Dans les modèles, la population des « Suisses d'origine » est considérée comme la modalité de référence. Les risques, présentés sous forme de risques relatifs (exponentielle du paramètre ß) pour l'un ou l'autre groupe, s'expriment par rapport à cette modalité, après prise en compte des facteurs de confusion. Un risque supérieur à l'unité signifie que la probabilité pour une personne du groupe considéré de présenter la caractéristique étudiée est supérieure à celle d'une personne du groupe de référence. Afin de mesurer la signification statistique des résultats, des intervalles de confiance à $95 \%$ et un degré de signification (test de p) sont présentés. Une valeur de p inférieure à 0,05 (indiquée dans les tableaux par un *) signifie qu'il y a moins de $5 \%$ de risques que le comportement de la modalité étudiée, estimé par l'enquête, ne soit en fait pas différent de celui de la modalité de référence, et plus de $95 \%$ de chances que la différence observée soit réelle. Les valeurs de $p$ inférieure à 0,01 et à 0,001 sont également indiquées dans les tableaux $\left({ }^{* *}\right.$ et $\left.{ }^{* * *}\right)$

\section{NOTES}

1. Cette étude a été réalisée dans le cadre du subside no 5004-66 389 du Fonds national pour la recherche scientifique, Berne, programme prioritaire « Demain la Suisse », que nous remercions. 2. Personne déclarant avoir une fonction dirigeante dans l'entreprise dans laquelle elle est active. 


\section{RÉSUMÉS}

Reposant sur des données du Panel Suisse des Ménages, cette étude dresse un tableau général des modes de vie et d'intégration professionnelle des communautés ou collectivités définies selon l'origine (nationalité des parents) et la nationalité actuelle. Elle montre les difficultés d'intégration structurelle observées par les différentes collectivités, en particulier celles originaires de certaines régions de l'Europe (Europe non communautaire et Europe du Sud). Ces difficultés peuvent certes être mises en relation avec le niveau de formation achevé, mais aussi avec des pratiques discriminatoires, qui conduisent entre autres à un taux de chômage élevé parmi les collectivités étrangères. Les disparités entre Suisses et populations d'origines étrangères se maintiennent après la naturalisation, les Suisses dont les parents étaient de nationalité étrangère au moment de la naissance présentant également un risque plus élevé de chômage, comparativement aux Suisses d'origine.

Professional Integration of Foreigners and of People of Foreign Origin in Switzerland. Using the Swiss Households Panel data, this study draws a general picture of the ways of life and professional integration of communities defined, according to the country of origin (nationality of the parents at birth) and the current nationality. It shows the difficulties of being integrated in a structural point of view among the various communities, in particular among those coming from some regions of Europa (non-EC countries and Southern Europe). These difficulties can certainly be linked with the level of education, but also with discriminatory practices, which lead to an increased rate of unemployment among the foreign communities. The disparities between the Swiss population and the one of foreign origin are maintained after naturalization : the group of Swiss people whose parents were of foreign nationality at birth also present a higher risk of unemployment, comparatively with persons of Swiss origin.

Integración profesional de la población suiza de origen y nacionalidad extranjeras. A partir de los datos del Panel de Hogares Suizos, este artículo presenta un abanico de los modos de vida y de la integración profesional de comunidades en función del origen (nacionalidad de los padres) y de la nacionalidad actual. El estudio demuestra las dificultades de integración estructural a las que se enfrentan ciertas comunidades, en particular aquellas originarias de ciertas regiones de Europa (Europa no comunitaria y del Sur). Estas dificultades pueden efectivamente deberse al nivel de formación, mas también a prácticas discriminatorias que conducen a una tasa de paro elevada entre los colectivos extranjeros. Las disparidades entre suizos y ciertas comunidades de origen extranjero se mantienen tras la naturalización. En comparación con los suizos de origen helvético, los suizos cuyos padres eran extranjeros cuando ellos nacieron presentan un riesgo de paro más elevado.

\section{INDEX}

Mots-clés : formation, insertion professionnelle, marché du travail Index géographique : Suisse 
AUTEUR

\section{PHILIPPE WANNER}

Forum suisse pour l'étude des migrations et de la population, Rue St-Honoré 2, CH-2000

Neuchâtel 